\title{
Aprendizaje en Antropología de la Comunicación a Través de Proyecto: los Secretos de "Assasin"s Creed IV:Black Flag" y "FIFA18" Descubiertos por Estudiantes
}

\author{
ÁNGELES CASTAÑo MADROÑAL \\ Universidad de Sevilla \\ Dpto. Antropología Social \\ acastamad@us.es \\ ORCID: https://orcid.org/000-0002-3617-8041 \\ D.O.I.: http://dx.doi.org/10.12795/JDU.2018.i01.102 \\ Pp.: 1811-1832
}

\section{Resumen}

Se presenta un Ciclo de Mejora Docente basado en aprendizaje a través de proyecto tomando como base el aprendizaje por resolución de problemas. Los contenidos estructurales se articulan procurando la aplicación de las teorías y métodos de análisis semiosemántico y simbólico propios de la antropología de la comunicación sobre dos videojuegos que forman parte del conocimiento del entorno de los estudiantes, como estudios de caso para experimentar este modelo de enseñanza-aprendizaje aplicado en la docencia universitaria. Se muestra el proceso desde el diseño de contenidos, las secuencias de 
actividades, el sistema de evaluación a partir de los conocimientos previos de los estudiantes, y el análisis de los resultados obtenidos y del modelo aplicado.

Palabras Clave: Antropología de la Comunicación, Grado en Antropología, docencia universitaria, experimentación docente universitaria, conocimiento del entorno.

\section{Breve descripción del contexto de intervención.}

Esta asignatura viene siendo objeto de mejora a través del programa de la FIDOP y la REFID desde 2015, de modo que se ha ido nutriendo de sucesivos ciclos de mejora que han permitido adaptar el programa docente en su totalidad, e ir encajando paulatinamente el modelo posible e ideal. Este es el 4o curso consecutivo en el programa de innovación docente de la asignatura Antropología de la Comunicación, una optativa de 2 o curso del Grado de Antropología Social. En esta ocasión se trata de un ciclo de 30 horas totales. Lo que equivale a 7 semanas de docencia más una sesión de dos horas en la semana 8 dedicada a la evaluación final de la enseñanza y aprendizaje. La docencia se articula en 2 sesiones de 2 horas por semana. Las sesiones se llevan a cabo, como en todos los cursos anteriores en un aula que tiene habilitados equipo informático y audiovisual, aunque infraestructuralmente la sala encajada entre los pilares de sostén y bóveda de una de las galerías del edificio monumental histórico (antigua Real Fábrica Tabacos) de la Universidad de Sevilla, tiene mala sonoridad y problemas de reverberación que dificultan mucho el disfrute de las abundantes actividades prácticas audiovisuales en esta asignatura.

El grupo matriculado este curso son 20 estudiantes. Pero el absentismo por casuísticas variadas, donde destaca el alto porcentaje de estudiantes becados con estancia en centros estatales, europeos y americanos, reduce el grupo a 9 presenciales que se acogen al sistema de evaluación y aprendizaje continuo establecido en la asignatura: 
3 proceden de las becas de intercambio con universidades norteamericanas (2 chicas, una de ellas con formación en biología, y otra de literatura española, y un chico neoyorkino estudiante de audiovisuales); 4 más y 1 chica son estudiantes autóctonos del título, y 1 joven chino con residencia de estudiante que cursa el título íntegramente en nuestra universidad. Como en cursos anteriores destaca la multiculturalidad y la multidisciplinariedad de la formación superior del grupo. El proyecto diseñado ha dado lugar a una alta participación y una dinámica de trabajo continuo en los dos equipos de trabajo, tanto en el aula presencialmente como fuera de ella, desarrollando búsquedas de información autónomas y creatividad aplicada de las teorías.

\section{Diseño de un CMD basado en proyecto.}

He optado por un proyecto de investigación como modelo de enseñanza-aprendizaje que se basa en el conocimiento del medio de los propios alumnos (Finkel, 2008) y en el aprendizaje por resolución de problemas (ARP), y los 7 principios básicos de Bain, que mantengo en mi modelo (Bain, 2007:113-132). Estas bases son las que mejor se adaptan a las capacidades actitudinales y procedimentales a entrenar en este nivel del título que estudian, y que permiten el aprendizaje participativo y autogestionado del discente llevando a la aplicación las principales teorías de análisis simbólico y semiosemántico más conocidos en la antropología de la comunicación. La estrategia es lograr una progresión que ambiciona que sean los propios estudiantes los que movilicen los contenidos a través de problemas-respuestas, y alcanzar una perspectiva general de los contenidos y sus aplicaciones posibles en los estudios de caso, para dar las respuestas más adecuadas. También para asentar la base de una asignatura compleja que ha de afrontar el dilema de que se ubica en un nivel 
introductorio del título. La articulación de lo aprendido en el FIDOP y la REFID se contiene en estos contenidos del modelo: 1) testado de los intereses de los estudiantes; 2) plantear un problema de investigación en este caso asociado a un proyecto de participación colectiva; 3 ) articular sus ideas en las cuestiones a resolver establecidos alrededor de subproblemas que estructuran los contenidos aplicables al proyecto; 4) diseño de las secuencias de actividades del proyecto; 5) preevaluación de saberes o conocimientos previos de los estudiantes entre las secuencias diseñadas para testar la evolución en su aprendizaje (de Alba, 2016); 6) elaboración de resultados del proyecto de investigación y transferencia de hallazgos y logros a los compañeros como sistema de enseñanza-aprendizaje entre pares; 7) posevaluación del aprendizaje; 8) posevaluación de la enseñanza; 9) analizar el ciclo de mejora para ir mejorando el modelo a partir de los obstáculos detectados. El modelo en su conjunto se basa en los progresos derivados del aprendizaje en el FIDOP (Porlan et al., 2017).

De acuerdo a este modelo, los propios estudiantes eligieron, en consonancia con sus inquietudes, que el objeto de estudio del proyecto serían videojuegos, y a partir de su conocimiento del medio, seleccionaron dos productos ampliamente comercializados como estudios de caso. El proyecto se desarrolló desde el 19 de marzo al 6 de junio, y ha conducido durante todo el cuatrimestre la articulación permanente entre teoría y práctica.

En relación al aprendizaje del medio, vengo articulando con los contenidos de la asignatura conferencias externas y actividades culturales que se dan en la US o en Sevilla entre febrero y junio, que es el calendario de la asignatura. En este sentido, el día 12 de marzo, acudieron a dos conferencias entre las 12.00 y las 14.00 en el Centro Internacional de la US sobre cine y flamenco que centraban contenidos del tema 4, 6 y 7 del temario. 
El modelo que he llevado a cabo queda sintetizado así:

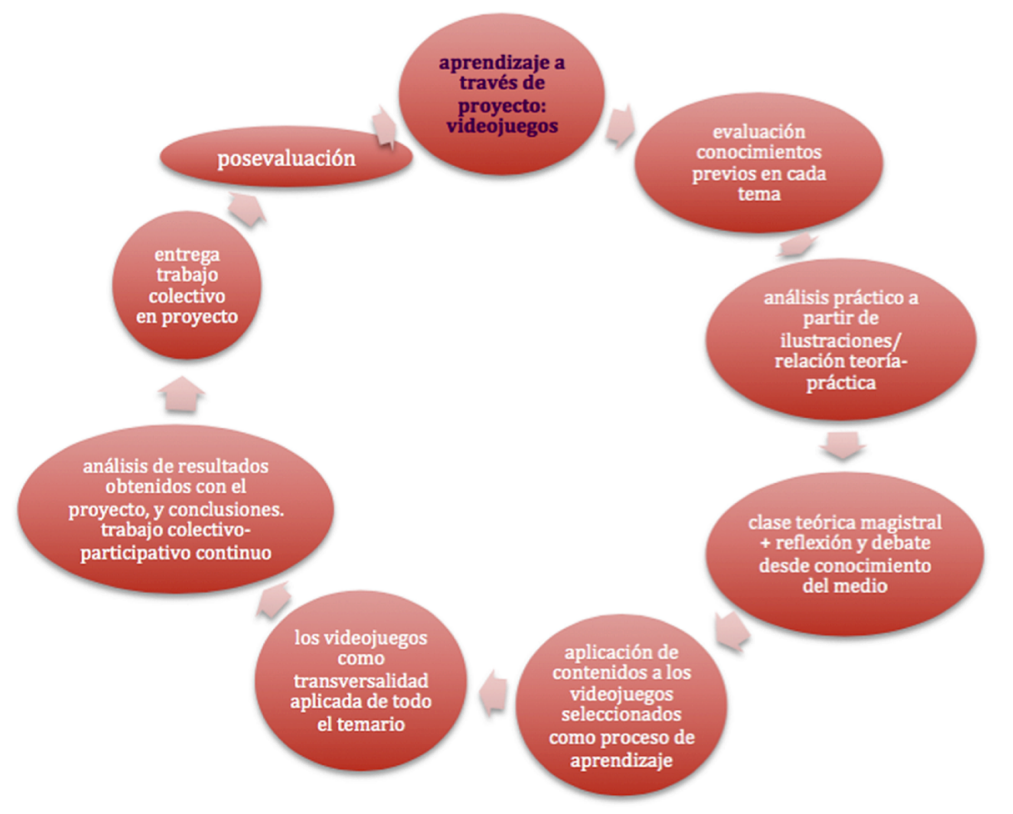

Figura 1. Modelo del CMD.

En este modelo basado en ARP a través de un proyecto de investigación colectivo que estudia los videojuegos, el objetivo es establecer feedback entre el conocimiento adquirido y circulado en el aula y el conocimiento del medio / producto de consumo con el que tienen una experiencia cotidiana, y colectiva generacional. Para ello, durante una sesión de 1 hora en la primera semana del curso, los estudiantes seleccionaron como estudios de caso 2 videojuegos que conocían bien, y se adaptaba a los contenidos a circular y aplicar en el curso. Tengo que aclarar, que en contra de mis propias inclinaciones por el cine como objeto de estudio trabajado en dos cursos anteriores. Es por ello, que tuve que adaptar y repensar las preguntas problemas y la articulación aplicada para que se pudiese llevar a cabo el proyecto de investigación colectivo.

Jornadas de Formación e Innovación Docente del Profesorado I № 1 (2018)

Esta obra se distribuye con la licencia Creative Commons 
El proyecto de investigación se mantiene de forma transversal a todo el temario, articulando los contenidos al proyecto de estudio, y permitiendo ver en un caso empírico lo que ha circulado a nivel teórico en el aula. También ha sido una novedad introducir primero la experimentación práctica a partir de los conocimientos de los estudiantes al inicio de cada actividad, para impartir después la teoría, y volver a realizar una práctica a posteriori en participación colectiva orientada, para incentivar la autoevaluación en los estudiantes y su aprendizaje personal contrastado. Se trata de ampliar la reflexión más allá del aula y trascender la teoría a la observación del comportamiento social y/o los productos culturales, como una cuestión fundamental para el aprendizaje de competencias y aptitudes propias de la antropología social desde los niveles más básicos del título.

\section{Mapa de contenidos, secuencias de actividades y problemas/preguntas-claves.}

El desarrollo del temario ha mantenido la transversalidad de contenidos docentes con el trabajo participativo y grupal en el proyecto de investigación, desde el tema 4 al 11. Ellos han trabajado a partir del análisis profundo realizado al unísono entre ambos estudios de caso. Un caso se ha trabajado en un equipo de 4 personas, y otro en equipo de 5 , y estableciendo una puesta en común en cada sesión dedicada al proyecto para transferir los aspectos analizados en cada videojuego dentro y fuera del aula. He ido conduciendo los contenidos que tenían que aplicar circulando los problemas/preguntas que tenían que resolver secuencialmente. De esta forma se ha ido construyendo mediante las prácticas en el aula un solo proyecto con dos estudios de caso. Debido a que el CDM de este año es de 30 horas se ha trabajado desde el tema 4 al 11. A partir del tema 4 se entra en una mayor profundización en las formas de la comunicación desde la diversidad cultural 
humana hasta las tecnologías de nuestra sociedad. La actividad de este año, transforma y mejora radicalmente casi la totalidad del temario de la asignatura, en cuanto el proyecto permite una traslación continua entre teoría y práctica todo el cuatrimestre.

En la ilustración destaco los conceptos y contenidos claves de los temas que se articulan con las dos fases de trabajo con el proyecto de los videojuegos, una más descriptiva en una primera fase, y otra posterior más analítica relacionada con cómo nos relacionamos con la tecnología mediática. En rojo he colocado las capacidades actitudinales generales de la asignatura a través del proyecto, y en verde las procedimentales generales de la asignatura y específicas e intelectuales del proyecto.

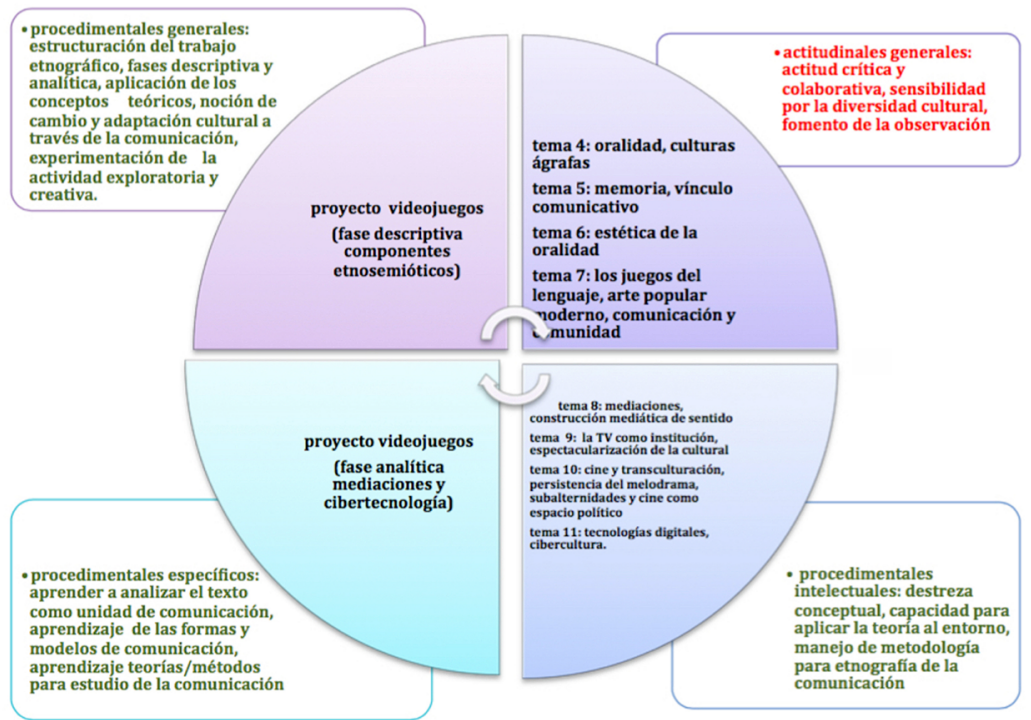

Figura 2. Mapa de contenidos.

A continuación se muestra la selección de preguntas clave y la articulación aplicada al proyecto de investigación, de modo que los contenidos teóricos aprendidos de forma reflexiva puedan experimentarse en su dimensión 

plado y abordado como texto. Para llevar a cabo este CMD se ha establecido una secuencia de trabajo en el aula en las 7 semanas indicadas antes más 1 sesión evaluadora final de 2 horas, de acuerdo al modelo que he diseñado.

\section{Tabla 1.}

Preguntas claves y programa semanal de sesiones

\begin{tabular}{|c|c|c|}
\hline Temporalidad & Tema 4 & Preguntas claves \\
\hline $\begin{array}{l}\text { Semana } 1 / \text { sesión } \\
1 \text { y sesión } 2\end{array}$ & Contenidos & $\begin{array}{l}\text { - La oralidad es específica de las culturas } \\
\text { ágrafas? } \\
\text { - La oralidad es un tipo de comunicación } \\
\text { más simple que otras? } \\
\text { - Hay una etnosemiótica específica en las } \\
\text { culturas ágrafas? } \\
\text { - La oralidad es sólo comunicación verbal? } \\
\text { - La oralidad es sólo propia del pasado y } \\
\text { de sociedades atrasadas o simples? }\end{array}$ \\
\hline \multirow[t]{2}{*}{$\begin{array}{l}\text { Semana 1/sesión } \\
2 \text { ( } 1 \text { hora) }\end{array}$} & PROYECTO & $\begin{array}{l}\text { * Identificación de los rasgos tipográficos, y } \\
\text { semióticos externos. } \\
\text { * Identificación aplicada de la oralidad en } \\
\text { los videojuegos. }\end{array}$ \\
\hline & Tema 5 & Preguntas claves \\
\hline $\begin{array}{l}\text { Semana 2/se- } \\
\text { sión } 1\end{array}$ & Contenidos & $\begin{array}{l}\text { - Existe una racionalidad expresivo-sim- } \\
\text { bólica específica en la diversas cultu- } \\
\text { ras o es un universal cultural? } \\
\text { - Qué relación hay entre la memo- } \\
\text { ria hiponoética, el aprendizaje y la } \\
\text { comunicación? } \\
\text { - Cómo se construye el vínculo comu- } \\
\text { nicativo en relación al sentido de } \\
\text { comunidad? }\end{array}$ \\
\hline \multirow[t]{2}{*}{$\begin{array}{l}\text { Semana 2/sesión } \\
2 \text { (1 hora) }\end{array}$} & PROYECTO & $\begin{array}{l}\text { * Trabajo aplicado de la construcción me- } \\
\text { diada de vínculo y comunidad en las tecno- } \\
\text { logías de videojuegos. De los signos externos } \\
\text { a los del círculo narrativo y sus lenguajes. }\end{array}$ \\
\hline & Tema 6 & Preguntas claves \\
\hline
\end{tabular}

Jornadas de Formación e Innovación Docente del Profesorado I № 1 (2018) Esta obra se distribuye con la licencia Creative Commons Reconocimiento-NoComercial-SinObraDerivada Internacional (CC BY-NC-ND 4.0.) 


\begin{tabular}{|c|c|c|}
\hline $\begin{array}{l}\text { Semana } 2 \text { /sesión } \\
1 \text { y sesión } 2\end{array}$ & Contenidos & $\begin{array}{l}\text { - Podrías identificar claves estéticas de la } \\
\text { comunicación verbal? } \\
\text { - Ilustra con ejemplos etnográficos mo- } \\
\text { dos de construir significados a través } \\
\text { enunciados propios de la estética ver- } \\
\text { bal, puedes recurrir a rituales y ma- } \\
\text { nifestaciones culturales de interés } \\
\text { etnográfico. }\end{array}$ \\
\hline \multirow[t]{2}{*}{$\begin{array}{l}\text { Semana } 2 \text { /sesión } \\
2 \text { ( } 1 \text { hora) }\end{array}$} & PROYECTO & $\begin{array}{l}\text { * Identificación de las claves estéticas en } \\
\text { la semiótica y los lenguajes propios del } \\
\text { videojuego. } \\
\text { * Identificación de símbolos usados en la es- } \\
\text { tética del videojuego }\end{array}$ \\
\hline & Tema 7 & Preguntas claves \\
\hline $\begin{array}{l}\text { Semana 3/se- } \\
\text { sión } 1\end{array}$ & Contenidos & $\begin{array}{l}\text { - qué relación guarda lo festivo con la } \\
\text { construcción y comunicación de } \\
\text { comunidad? } \\
\text { - Identifica elementos específicos andalu- } \\
\text { ces propios del arte popular moderno. } \\
\text { - Qué características presenta estos ele- } \\
\text { mentos desde la perspectiva de la co- } \\
\text { municación y desde sus lenguajes } \\
\text { específicos? }\end{array}$ \\
\hline \multirow[t]{2}{*}{$\begin{array}{l}\text { Semana } 3 / \text { se- } \\
\text { sión } 2 \text { (1 hora) }\end{array}$} & PROYECTO & $\begin{array}{l}\text { * Análisis de las semejanzas entre los recur- } \\
\text { sos estéticos en las performances interacti- } \\
\text { vas de los videojuegos, con los recursos del } \\
\text { arte popular moderno. } \\
\text { * Modo en que el videojuego produce una } \\
\text { estética comunicativa específica de una } \\
\text { comunidad. }\end{array}$ \\
\hline & Tema 8 & Preguntas claves \\
\hline $\begin{array}{l}\text { Semana 4/se- } \\
\text { sión } 1\end{array}$ & Contenidos & $\begin{array}{l}\text { - Sabrías explicar que diferencia la } \\
\text { oralidad de las mediaciones de } \\
\text { comunicación? } \\
\text { - Crees que hay una relación entre los } \\
\text { procesos de identificación y el espa- } \\
\text { cio mediado como generador de iden- } \\
\text { tidades/identificaciones? Explícalo } \\
\text { con ejemplos del medio. } \\
\text { - Cómo se genera un imaginario mediá- } \\
\text { tico? Podemos ejemplificar a través de } \\
\text { nuestra experiencia directa? } \\
\text { - Puede ser el sentido de realidad común } \\
\text { una producción? }\end{array}$ \\
\hline
\end{tabular}

Jornadas de Formación e Innovación Docente del Profesorado | № 1 (2018) Esta obra se distribuye con la licencia Creative Commons Reconocimiento-NoComercial-SinObraDerivada Internacional (CC BY-NC-ND 4.0.) 


\begin{tabular}{|c|c|c|}
\hline $\begin{array}{l}\text { Semana 4/sesión } \\
2 \text { (1hora) }\end{array}$ & PROYECTO & $\begin{array}{l}\text { * Análisis de los videojuegos y su producción } \\
\text { de sentido, en claves de género, clase social, } \\
\text { etnia-nación, edad. } \\
\text { * Circulaciones de sentido a través del vi- } \\
\text { deojuego: las comunidades gamer. }\end{array}$ \\
\hline & Tema 9 & Preguntas claves \\
\hline $\begin{array}{l}\text { Semana 4/sesión } \\
1 \text { y } 2\end{array}$ & Contenidos & $\begin{array}{l}\text { - Porqué el poder mediático es un asunto } \\
\text { político?; qué relación guardan ambos } \\
\text { espacios en la sociedad tecnológica? } \\
\text { - El melodrama puede ser un estilo cultu- } \\
\text { ral histórico en la comunicación y sus } \\
\text { producciones? } \\
\text { - Relación entre el continuum cultural del } \\
\text { melodrama como estética comunica- } \\
\text { tiva y las interfaces de las comunica- } \\
\text { ción que plantea Manovich. } \\
\text { - Consumes lo que se proyecta y circula } \\
\text { en los media?. } \\
\text { - Relación entre construcciones del sen- } \\
\text { tido y consumo. }\end{array}$ \\
\hline \multirow[t]{2}{*}{$\begin{array}{l}\text { Semana 5/sesión } \\
2 \text { ( } 1 \text { hora) }\end{array}$} & PROYECTO & $\begin{array}{l}\text { * Análisis del contenido narrativo, melo- } \\
\text { drama, tragicomedia y sátira, significados y } \\
\text { semántica de la narración (aplicación de pa- } \\
\text { limpsesto, intertextualidad, semiosfera, he- } \\
\text { teroglosia y cronotopos como conceptos } \\
\text { aplicables a los videojuegos) }\end{array}$ \\
\hline & Tema 10 & Preguntas claves \\
\hline $\begin{array}{l}\text { Semana 5/se- } \\
\text { sión } 1\end{array}$ & Contenidos & $\begin{array}{l}\text { el poder de la imagen en las culturas ur- } \\
\text { banas establece alguna relación en- } \\
\text { tre los distintos productos culturales de } \\
\text { comunicación? } \\
\text { Cómo pueden las nuevas tecnologías cons- } \\
\text { truir espacios políticos para los distintos } \\
\text { grupos y clases sociales? } \\
\text { Sabes lo que es la voz como poder en el es- } \\
\text { pacio político? } \\
\text { Qué quiere decir García-Canclini cuando } \\
\text { considera nuestra sociedad como "cultura } \\
\text { de la imagen"? } \\
\text { Qué relación guarda el cine con la cultura } \\
\text { global urbana y por otro lado, con los movi- } \\
\text { mientos artísticos glocales? }\end{array}$ \\
\hline
\end{tabular}

Jornadas de Formación e Innovación Docente del Profesorado | № 1 (2018) Esta obra se distribuye con la licencia Creative Commons Reconocimiento-NoComercial-SinObraDerivada Internacional (CC BY-NC-ND 4.0.) 


\begin{tabular}{|c|c|c|}
\hline $\begin{array}{l}\text { Semana 5/sesión } \\
2 \text { (1 hora) }\end{array}$ & PROYECTO & $\begin{array}{l}\text { * Análisis de la imagen, la publicidad y la } \\
\text { cultura del cine en la tecnología de los vi- } \\
\text { deojuegos. Recursos usados. } \\
\text { * Narrativa, nudo, desenlace en los juegos. }\end{array}$ \\
\hline & Tema 11 & Preguntas claves \\
\hline $\begin{array}{l}\text { Semana 6/se- } \\
\text { sión } 1\end{array}$ & Contenidos & $\begin{array}{l}\text { Porqué Escobar considera que estamos en la } \\
\text { edad de la cibercultura? } \\
\text { Relación de las nuevas tecnologías con los } \\
\text { ámbitos público/privado, íntimo/extimo. } \\
\text { Las redes digitales reproducen sentidos so- } \\
\text { ciales de identificación? Cómo se construyen } \\
\text { estos sentidos? } \\
\text { La cibernética, los cyborg y la informática } \\
\text { guardan una estrecha relación con la cog- } \\
\text { nición humana. Puedes avanzar alguna } \\
\text { apreciación desde lo aprendido entre me- } \\
\text { diaciones y sentido? }\end{array}$ \\
\hline $\begin{array}{l}\text { Semana 6/sesión } \\
2 \text { (1 hora) }\end{array}$ & PROYECTO & $\begin{array}{l}\text { * Descripción y análisis de las emociones en } \\
\text { la interacción gamer en los videojuegos. } \\
\text { * Análisis de las emociones generadas en } \\
\text { la interacción con el videojuego. Procesos } \\
\text { cognitividad-afectos. } \\
\text { * Análisis de los videojuegos como producto } \\
\text { de las industrias culturales y su potencia- } \\
\text { lidad para impulsar emociones y construir } \\
\text { sentido. } \\
\text { * Relación entre las industrias culturales } \\
\text { como fabricantes de emociones y la noción } \\
\text { de pantalla global de Lipovetsky. }\end{array}$ \\
\hline $\begin{array}{l}\text { Semana } 7 / \text { se- } \\
\text { sión } 1\end{array}$ & PROYECTO & $\begin{array}{l}\text { * Generación y estructura de un documento } \\
\text { y texto colectivo de análisis y resultados }\end{array}$ \\
\hline $\begin{array}{l}\text { Semana } 7 / \text { se- } \\
\text { sión } 2\end{array}$ & PROYECTO & $\begin{array}{l}\text { * Presentación y defensa de los resultados } \\
\text { del proyecto de estudio. }\end{array}$ \\
\hline $\begin{array}{l}\text { Semana } 8 / \text { sesión } \\
1 \text { ( } 2 \text { horas) }\end{array}$ & EVALUACIÓN & $\begin{array}{l}\text { AUTOEVALUACIÓN DEL ALUMNO/ EVALUA- } \\
\text { CIÓN DE LA DOCENCIA/EVALUACIÓN DEL } \\
\text { APRENDIZAJE }\end{array}$ \\
\hline
\end{tabular}

La racionalidad temporal de las sesiones queda estructurada como se ve en la tabla siguiente, de forma que tenga cabida la pre y posevaluación de los aprendizajes, y de acuerdo al inicio y fin de los temas impartidos. 
Tabla 2.

Secuencia de actividades en el aula

\begin{tabular}{|l|l|}
\hline Sesiones X semana & Actividades \\
\hline 1ó sesión de 2 horas & * 20' preevaluación \\
& * 40' análisis aplicado participativo a partir de \\
& videos \\
& *60' desarrollo teoría a partir de 1 lectura comen- \\
& tada participativa + clase magistral \\
\hline 2o- sesión de 2 horas & $\begin{array}{l}\text { * 40' análisis aplicado participativo en videos } \\
\text { *60' práctica colectiva y grupal en el proyecto de } \\
\text { investigación }\end{array}$ \\
& *20' posevaluación \\
\hline
\end{tabular}

El proyecto de investigación requiere que se articule el mapa de contenidos de la figura 1 respecto de las preguntas problemas aplicables de acuerdo al programa temporal de sesiones en la tabla 1. Para tener una visión de conjunto que articule el proyecto en la actividad docente a lo largo de todo el curso y los contenidos del temario oficial aprobado por el Departamento de Antropología según establece la normativa vigente en la Facultad de Geografía e Historia, en la siguiente ilustración (figura 3) se muestra la lógica que se ha diseñado. Siendo la flecha el proyecto de investigación en su transversalidad a lo largo del trabajo en el aula durante 1 hora cada semana hasta el 6 de junio, se refleja la secuencia de contenidos y temas entre los bloques temáticos con aquellos contenidos más imbricados entre sí desde que se inicia el curso hasta el final. El proyecto establece un vínculo aplicado de todo el temario. 


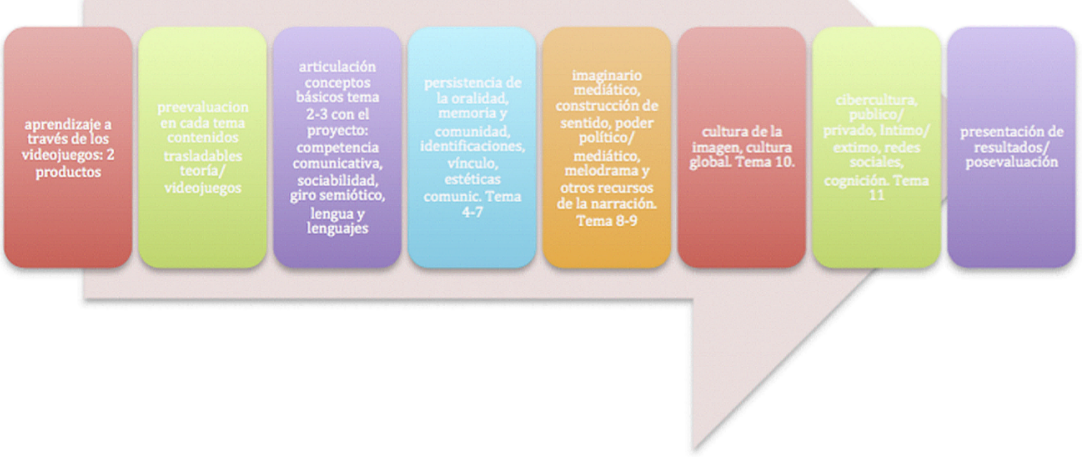

Figura 3: Transversalidad del proyecto respecto de los contenidos.

\section{Cuestionario inicial y final para seguimiento del aprendizaje.}

La evaluación del proceso de aprendizaje de acuerdo a la dinámica de clase se ha testado con un cuestionario de pre y pos evaluación que toma en cuenta el modelo de escalera de aprendizaje (de Alba, 2016). Las preguntas que se han barajado se encuentran en la tabla 1 de las preguntas claves y la temporalidad semanal de contenidos. Pero también se realiza una evaluación final de la enseñanza el último día y después del cuestionario de posevaluación de conocimientos.

Tabla 3.

Posevaluación de la enseñanza:

\begin{tabular}{|c|c|c|}
\hline & $\begin{array}{l}\text { consideras que la metodología de en- } \\
\text { señanza/aprendizaje te ha ayudado a } \\
\text { comprender y aprehender los conteni- } \\
\text { dos del temario? }\end{array}$ & $\begin{array}{l}\square \text { no. } \\
\square \text { en parte, pero es } \\
\text { mejorable. } \\
\square \text { si y estoy muy satisfecho. }\end{array}$ \\
\hline & crees que podría mejorarse el método? & $\begin{array}{l}\square \text { no estoy satisfecho, y me } \\
\text { ha confundido más que } \\
\text { aclarado. } \\
\square \text { es mejorable. } \\
\square \text { ha estado muy bien así. }\end{array}$ \\
\hline
\end{tabular}




\begin{tabular}{|l|l|}
\hline $\begin{array}{l}\text { 3.- qué contenidos te han resultado más } \\
\text { complejos o consideras que no has asimi- } \\
\text { lado bien? }\end{array}$ & Comentario libre \\
\hline $\begin{array}{l}\text { 4.- si tú fueses el que tiene que explicar } \\
\text { esta asignatura, cómo lo habrías hecho? }\end{array}$ & Comentario libre \\
\hline $\begin{array}{l}\text { 5.- si crees que hay alguna forma de mejo- } \\
\text { rar los contenidos y las prácticas, podrías } \\
\text { ayudarme si me dices cómo. }\end{array}$ & Comentario libre \\
\hline $\begin{array}{l}\text { 6.- señala que crees que ha faltado, o } \\
\text { qué esperabas haber aprendido y no ha } \\
\text { ocurrido. }\end{array}$ & Comentario libre \\
\hline $\begin{array}{l}\text { 7.- califica con una nota media el modelo de } \\
\text { enseñanza }\end{array}$ & Calificación numérica 1-10 \\
\hline $\begin{array}{l}\text { 8.- califica con una nota media la ense- } \\
\text { ñanza impartida por la profesora }\end{array}$ & Calificación numérica 1-10 \\
\hline
\end{tabular}

A lo largo del curso se ha llevado a cabo un diario de clases (Porlán, 2008) en el que se ha ido anotando las observaciones del día a día de la docencia y el proceso de enseñanza-aprendizaje en el aula. El objetivo es observar y poder rescatar las observaciones destacadas a lo largo del curso, y de alguna manera, realizar una investigación del propio hacer personal como docente.

\section{Aplicación del Ciclo de Mejora Docente.}

\section{Relato resumido de las sesiones.}

De acuerdo a este diseño, las sesiones se encontraban estructuradas de modo que en aquellas que coincidían con el inicio de un nuevo tema, se realizaba primero la preevaluación. La posevaluación se realizaba en la sesión en que finalizaba los contenidos del tema. De forma invariable, en todas las sesiones justo antes de iniciar los contenidos nuevos correspondientes se recapitulaba, a modo de recordatorio, las ideas principales de la sesión anterior en 5 minutos. Hay que tener en cuenta que se hace necesario 
debido a que las sesiones de la semana no son consecutivas, ya que la primera se hace en lunes, y la segunda en miércoles. Las sesiones de trabajo semanal durante 1 hora en el aula para aplicación en el proyecto, se realizaban los miércoles en la segunda hora de la sesión, ya que al ser la última asignatura del día, alguna vez se pudo alargar 5 minutos más si se había producido algún retraso. Esta circunstancia y conveniencia, fue consensuada con los estudiantes al empezar el curso. Hay que tener en cuenta también, que era el momento en que se ponía en común el trabajo realizado fuera del aula por cada equipo de trabajo. Ello implica que al final de cada sesión aplicada, los miércoles, se llevaba a cabo una previsión de tareas encargadas y por adelantado para que organizaran el trabajo fuera del aula, de cara a la sesión del miércoles siguiente en el proyecto. Sin el trabajo fuera del aula de los equipos, habría sido imposible iniciarles en el análisis de la comunicación a través de un proyecto aplicado. Ellos fueron conscientes de esta necesidad en el acuerdo establecido al inicio del curso, cuando asumieron este método de evaluación continua y de enseñanza-aprendizaje aplicado.

Las sesiones práctico-teóricas se establecía los lunes, y se procedía con 5 minutos de recapitulación, tras la preevalucación correspondiente de 15/20 minutos. Luego se llevaba a cabo un ensayo práctico basado en la intuición de los estudiantes sobre alguna fuente audiovisual corta que podía durar entre 20/25 minutos, seguido de la impartición teórica con referencias conceptos/autores/monografías al final de la sesión 15/20 minutos que se volvían a retomar en la primera hora de la sesión segunda de los miércoles, si se hacía necesario, para completar o culminar lo que quedase pendiente. Posteriormente se dejaba unos 20 minutos tras la teoría para ver sobre el video trabajado desde la intuición, lo que era necesario mejorar y poner de relieve lo que se había realizado correctamente o era necesario mejorar. Inmediatamente después de las sesiones anotaba mis observaciones en el diario de clases en unos 
10 minutos. Aunque en un par de ocasiones, fue tan deslumbrante el hallazgo de los propios estudiantes sobre sus estudios de caso, que procedi a anotar en el mismo aula para ser fiel al suceso y a las emociones desencadenadas, tanto en ellos como en mí misma.

\section{Evaluación del aprendizaje de los estudiantes.}

Quiero destacar aquí esas dos anotaciones del diario de clases, relacionadas con un par de los deslumbramientos colectivos ante los descubrimientos de los propios estudiantes sobre las técnicas de los videojuegos para generar emociones desde impresiones psico-sensitivas en el "gamer", a través de la tecnología de la imagen. Hallazgos resultado de aplicar los métodos y teorías.

" es sorprendente!!. Acaban de aplicarme en el aula un análisis secuencial y del lenguaje multimodal básico sobre el videojuego Assasin's (...) han identificado qué recursos simbólicos en la narrativa establecen 3 mundos que representan la desigualdad de las culturas y Estados del mundo (10 mundo/3o mundo), y me explican que reproduce el imaginario occidental del sistema/mundo"

"Ha explicado Manu en la pizarra su análisis de un recurso estético y técnico apasionante, que corta los 3 mundos paralelos del videojuego (el anodino mundo laboral/la entrada al videojuego/el apasionante mundo ficticio): un juego entre interfaces (aplican la lectura de Manovich). En el mundo laboral de una vida de autómata el gamer se ve así mismo de cuerpo entero en un universo gris y plano, con simulación propia del cine l en el corte al portal el ojo de la cámara se encuentra sobre los ojos del jugador y el gamer puede ver solo los mandos y sus manos / en el mundo ficticio, el ojo de dios, y viven y ven todo controlando todas las dimensiones y en una primera persona que es inmortal o puede vivir muchas vidas. Les explico a raíz de estos descubrimientos que hay recursos del cine posestructuralista, y que actúa un recurso técnico y estético de panoptismo muy usado en la literatura y el cine. Todos estamos muy excitados con el hallazgo!!"

Uno de los aspectos más emocionantes de este proyecto, es que tanto ellos como yo hemos aprendido en un proceso colectivo, que ha transformado las dinámicas 
en el aula y nuestras interacciones en una comunidad de aprendizaje bastante horizontal. No en vano, les confesé desde el principio mi preferencia por el cine, por mi desconocimiento experiencial en el consumo de videojuegos. El establecimiento de un grupo de Facebook para subir imágenes de los videojuegos que querían trabajar, esquemas de sus análisis, y otros comentarios permitió traer al aula de manera audiovisual sus trabajos desarrollados fuera: "Antropocomunicación US." También trabajaron en un texto escrito a varias manos en google-doc que cada equipo construyó, montando estructuradamente lo que habían ido realizando a lo largo del curso, y que luego volcaron en sendos power point que mostraron en la sesión 14 cuando cada equipo expuso su trabajo completo y finalizado para poder compartirlo y ser evaluado. Ellos eligieron el modo y la estética que consideraron más adecuada para dicha exposición, y se organizaron en equipos para definir los contenidos y el orden de exposiciones.

A continuación muestro la escalera de aprendizaje resultante de la fusión de las 6 pre y posevaluaciones realizadas, he decidido mostrarlo en unidades, ya que son sólo 9 alumnos:

Jornadas de Formación e Innovación Docente del Profesorado | № 1 (2018) Esta obra se distribuye con la licencia Creative Commons 
Tabla 4.

Resultados de la pre y posevaluación del aprendizaje

\begin{tabular}{|c|c|c|c|c|c|c|c|}
\hline \multirow[t]{7}{*}{ Cuestionario 1} & \multirow[t]{2}{*}{ preguntas } & \multicolumn{2}{|c|}{ Nivel 1} & \multicolumn{2}{|c|}{ Nivel 2} & \multicolumn{2}{|c|}{ Nivel 3} \\
\hline & & Pre & Pos & Pre & Pos & Pre & Pos \\
\hline & 1 & 9 & 0 & 0 & 6 & 0 & 3 \\
\hline & 2 & 9 & 0 & 0 & 2 & 0 & 7 \\
\hline & 3 & 5 & 0 & 5 & 3 & 0 & 6 \\
\hline & 4 & 9 & 0 & 0 & 2 & 0 & 7 \\
\hline & 5 & 7 & 0 & 2 & 4 & 0 & 5 \\
\hline \multirow[t]{8}{*}{ Cuestionario 2} & 1 & 0 & 0 & 5 & 3 & 4 & 7 \\
\hline & 2 & 8 & 0 & 1 & 5 & 0 & 4 \\
\hline & 3 & 9 & 1 & 0 & 5 & 0 & 3 \\
\hline & 4 & 7 & 1 & 2 & 4 & 0 & 4 \\
\hline & 5 & 9 & 0 & 0 & 3 & 0 & 6 \\
\hline & 6 & 1 & 1 & 6 & 4 & 2 & 4 \\
\hline & 7 & 7 & 0 & 2 & 3 & 0 & 6 \\
\hline & 8 & 6 & 2 & 3 & 4 & 0 & 3 \\
\hline \multirow[t]{4}{*}{ Cuestionario 3} & 1 & 0 & 0 & 5 & 3 & 4 & 6 \\
\hline & 2 & 0 & 0 & 4 & 2 & 5 & 7 \\
\hline & 3 & 2 & 0 & 6 & 5 & 1 & 4 \\
\hline & 4 & 1 & 0 & 6 & 2 & 3 & 7 \\
\hline \multirow[t]{5}{*}{ Cuestionario 4} & 1 & 2 & 0 & 7 & 4 & 0 & 5 \\
\hline & 2 & 5 & 1 & 4 & 4 & 0 & 5 \\
\hline & 3 & 5 & 2 & 4 & 3 & 0 & 5 \\
\hline & 4 & 0 & 0 & 6 & 2 & 3 & 7 \\
\hline & 5 & 1 & 0 & 7 & 3 & 1 & 6 \\
\hline
\end{tabular}

Jornadas de Formación e Innovación Docente del Profesorado | № 1 (2018) cc) (i) (2) Esta obra se distribuye con la licencia Creative Commons Reconocimiento-NoComercial-SinObraDerivada $\quad 4.0$ Internacional (CC BY-NC-ND 4.0.) 


\begin{tabular}{|c|c|c|c|c|c|c|c|}
\hline \multirow{4}{*}{ Cuestionario 5 } & 1 & 1 & 1 & 6 & 2 & 2 & 6 \\
\cline { 2 - 8 } & 2 & 1 & 1 & 5 & 2 & 3 & 6 \\
\cline { 2 - 8 } & 3 & 1 & 1 & 6 & 2 & 2 & 6 \\
\cline { 2 - 8 } & 4 & 0 & 0 & 6 & 2 & 3 & 7 \\
\cline { 2 - 8 } & 5 & 0 & 0 & 7 & 4 & 2 & 5 \\
\hline \multirow{6}{*}{ Cuestionario 6 } & 1 & 0 & 0 & 6 & 3 & 3 & 6 \\
\cline { 2 - 8 } & 2 & 2 & 1 & 5 & 3 & 2 & 5 \\
\cline { 2 - 8 } & 3 & 0 & 0 & 6 & 3 & 3 & 6 \\
\cline { 2 - 8 } & 4 & 0 & 0 & 8 & 2 & 1 & 7 \\
\cline { 2 - 8 } & 5 & 0 & 0 & 7 & 4 & 1 & 5 \\
\hline
\end{tabular}

En general se puede observar una mejora de todos en las posevaluaciones, incrementándose significativamente el número de alumnos que alcanzan el nivel superior 3. También es de destacar que conforme fueron realizando los cuestionarios fueron habituándose a la actividad, de modo que mientras en los dos primeros cuestionarios de preevaluación apenas explicaban ni desarrollaban sus afirmaciones, en los siguientes, pude observar cómo dedicaban un mayor detalle a la explicaciones razonadas de sus afirmaciones, a ilustrar con ejemplos, e incluso en algunos casos destacados referenciando las lecturas realizadas. Otro aspecto a destacar es que más de la mitad de ellos (6 de 9), terminaron articulando las respuestas aumentando la complejidad de sus razonamientos conforme se alcanzaron los 2 últimos cuestionarios de posevaluación; evidencia de que habían comprendido la complejidad acumulativa de los contenidos, y que éstos estaban imbricados a lo largo de todo el proceso.

Y este es el resultado de la evaluación que los estudiantes han realizado de la enseñanza y el CDM: 
Tabla5.

Evaluación final de la enseñanza y el CDM.

\begin{tabular}{|c|c|c|c|}
\hline Preguntas & $\operatorname{Res}$ & & \\
\hline 1 & 0 & 3 & 6 \\
\hline 2 & 0 & 3 & 6 \\
\hline 3 & $3 c$ & $\mathrm{SCC}$ & \\
\hline 4 & $\begin{array}{l}2 \mathrm{cc} \\
\mathrm{me}\end{array}$ & acti & $\begin{array}{l}\text { más la teoría e incre- } \\
\text { actividades externas }\end{array}$ \\
\hline 5 & $\begin{array}{l}2 \mathrm{cc} \\
\text { par }\end{array}$ & : al & er aun más espacio \\
\hline 6 & $\begin{array}{l}1 \mathrm{nc} \\
\mathrm{dic}\end{array}$ & dic & $\begin{array}{l}\text { tada para opinar; } 7 \\
\text { que no esperaban }\end{array}$ \\
\hline 7 & $3=7$ & 12 & \\
\hline 8 & $2=7$ & & \\
\hline
\end{tabular}

En general hay bastante satisfacción con el CDM y mi trabajo continuo con ellos. Tengo que destacar que las calificaciones más altas y el mayor grado de satisfacción lo han demostrado en el cuestionario los estudiantes norteamericanos. Los autóctonos eran más críticos, pero muy imprecisos a la hora de indicar mejoras. Dos de las estudiantes norteamericanas vinieron a despedirse terminada la docencia y antes de regresar a su país, expresando verbalmente lo interesante que les había resultado el proyecto y que habían aprendido gran cantidad de contenidos y aplicaciones a elementos del entorno que desconocían por su trayectoria formativa previa.

Un estudiante español con beca de movilidad internacional en una universidad mejicana, que este año cursa tercero del Grado, se ha ofrecido a dejarme un video grabado de 30 minutos explicando a los estudiantes del futuro curso, lo aprendido y los hallazgos en el videojuego Assasin's Creed que han analizado. Y dos de los estudiantes que se encuentran en 3 o cursando la asignatura de Métodos y Técnicas de Investigación en la que deben de diseñar y poner en práctica un proyecto, están trabajando juntos sobre los videojuegos. 


\section{Evaluación del diseño puesto en práctica.}

Esta forma de iniciar la asignatura tiene la ventaja de hacer la docencia flexible, y un contexto incentivador en la relación alumnos-profesora ya que cada año los intereses de los estudiantes introducen modificaciones en el CMD. Aunque ello haga necesario adaptar el programa y la teoría prevista. Mientras la pre-y posevaluación permite ir modulando los contenidos y el ritmo a los tiempos de aprendizaje, así como testar la necesidad de ir formulando y adaptando las secuencias y el ciclo participativo de preguntas-reflexiones-debate participativo, para ayudarles en la elaboración conjunta de respuestas, y unas conclusiones a partir de lo que han aprendido en las clases.

Si bien es cierto que dejar a los estudiantes guiar la enseñanza de acuerdo a sus gustos e intereses, me han obligado a un mayor esfuerzo de adaptación de contenidos y reorientación del CDM, tengo que decir que ha sido muy satisfactorio también para mí. Siento que he aprendido cosas que no hubiesen sido posible sin haber dado la oportunidad de cambiar mis previsiones y planes. Es por esto, que voy a mantener la dinámica de ofrecer en la primera semana del nuevo curso la oportunidad de desarrollar la aplicación de los contenidos en la temática o el ámbito de interés que ellos tengan. Siempre buscando el consenso del grupo, y de cara a desarrollar dinámicas de trabajo en equipo y aprendizaje colectivo y horizontal.

Considero que los principios didácticos argumentados en el CDM son los que permiten un abordaje adecuado al nivel del Grado que tienen los alumnos, y las capacidades que se pueden entrenar y desarrollar. Los 7 principios de Bain (Bain, 2007), el ARP, tomar en cuenta los modelos mentales de los estudiantes, su conocimiento del medio, y evaluar el proceso de aprendizaje contemplando una escalera progresiva (Bain, 2007; de Alba, 2016; Porlán et al., 2017) sientan los pilares de un modelo que ha mejorado sustancialmente mi experiencia docente y que considero 
asentado. Por eso, tras 4 años de proceso de innovación docente, creo que el modelo posible e ideal prácticamente se han fusionado.

Ciertamente, como los propios estudiantes piden, tengo la intención de incrementar las prácticas fuera del aula, y aprovechar las actividades formativas universitarias externas a la propia disciplina $u$ otras afines, que tengan que ver con los contenidos a aprender.

Mi objetivo en los próximos cursos se orientan a dos fines: mantener este modelo en antropología de la comunicación, y extender lo aprendido a las asignaturas de las 3 titulaciones en las que imparto docencia.

\section{Referentes bibliográficos}

Bain, K. (2007) Lo que hacen los mejores profesores universitarios. València: PUV.

De Alba, N. (2016) El análisis de los modelos mentales de los estudiantes antes y después de la docencia: utilidad y procedimiento. 2o seminario formativo presencial de la REFID.

Finkel, D. (2008): Dar clase con la boca cerrada. Valencia, España: PUV.

Porlán, R. (Coord.) (2017) Enseñanza universitaria. Cómo mejorarla. Madrid: Ediciones Morata.

Porlán, R. (2008) El diario de clase y el análisis de la práctica. Averroes. Red Telemática Educativa de Andalucía. Open acces: https://idus.us.es/xmlui/bitstream/ handle/11441/25448/El\%20Diario\%20de\%20clase\%20 y\%20el\%20análisis\%20de\%20la\%20 práctica..pdf?sequence=1. Acceso: 12/10/2018

Jornadas de Formación e Innovación Docente del Profesorado | № 1 (2018) Esta obra se distribuye con la licencia Creative Commons 